Published in final edited form as:

Gynecol Oncol. 2014 March ; 132(3): 585-592. doi:10.1016/j.ygyno.2014.01.015.

\title{
Temsirolimus with or without Megestrol Acetate and Tamoxifen for Endometrial Cancer: a Gynecologic Oncology Group Study
}

\author{
Gini F. Fleming ${ }^{1}$, Virginia L. Filiaci ${ }^{2}$, Brandon Marzullo ${ }^{2}$, Richard J. Zaino ${ }^{3}$, Susan A. \\ Davidson $^{4}$, Michael Pearl ${ }^{5}$, Vicky Makker ${ }^{6}$, James J. Burke II ${ }^{7}$, Susan L. Zweizig ${ }^{8}$, Linda \\ Van Le ${ }^{9}$, Parviz Hanjani ${ }^{10}$, Gordon Downey ${ }^{11}$, Joan L. Walker ${ }^{12}$, Henry D. Reyes ${ }^{13}$, and \\ Kimberly K. Leslie ${ }^{13}$ \\ ${ }^{1}$ University of Chicago, Chicago, IL 60637 \\ ${ }^{2}$ Gynecologic Oncology Group Statistical \& Data Center, Roswell Park Cancer Institute, Buffalo, \\ NY 14263 \\ ${ }^{3}$ Hershey Medical Center, Medical Center of Pennsylvania State University, Hershey, PA 17033 \\ ${ }^{4}$ University of Colorado Cancer Center - Anschutz Cancer Pavilion, Aurora, CO 80045 \\ ${ }^{5}$ Stony Brook University Hospital, Stony Brook, NY 11794 \\ ${ }^{6}$ Memorial Sloan-Kettering Cancer Center, New York, NY 10021 \\ ${ }^{7}$ Memorial Medical Center, Savannah, GA 31404 \\ ${ }^{8} \mathrm{U}$ Mass Memorial Medical Center, Worcester, MA 01605 \\ 9UNC-Chapel Hill, Chapel Hill, NC 27599 \\ ${ }^{10}$ Hanjani Institute for Gynecologic Oncology, Abington, PA 19001 \\ ${ }^{11}$ Gynecologic Oncology of West Michigan, Grand Rapids, MI 49546 \\ ${ }^{12}$ University of Oklahoma, Oklahoma City, OK 73190 \\ ${ }^{13}$ University of lowa Hospitals and Clinics, lowa City, IA 52242
}

\begin{abstract}
Background-Preclinical evidence suggested that blockade of the PI3K/AKT/mTOR pathway might overcome resistance to hormonal therapy.

Methods-We performed a randomized phase II trial of intravenous temsirolimus $25 \mathrm{mg}$ weekly versus the combination of weekly temsirolimus with a regimen of megestrol acetate $80 \mathrm{mg}$ bid for
\end{abstract}

\footnotetext{
(C) 2014 Elsevier Inc. All rights reserved.

Corresponding Author: Gini F. Fleming, MD, University of Chicago, Medical Oncology, 5841 South Maryland Avenue, MC 2115, Chicago, IL 60637, Telephone: 773-702-6712, Fax: 773-702-0963, gfleming@medicine.bsd.uchicago.edu.

CONFLICT OF INTEREST

The authors wish to declare that there are no conflicts of interest.

Publisher's Disclaimer: This is a PDF file of an unedited manuscript that has been accepted for publication. As a service to our customers we are providing this early version of the manuscript. The manuscript will undergo copyediting, typesetting, and review of the resulting proof before it is published in its final citable form. Please note that during the production process errors may be discovered which could affect the content, and all legal disclaimers that apply to the journal pertain.
} 
three weeks alternating with tamoxifen $20 \mathrm{mg}$ bid for three weeks in women with recurrent or metastatic endometrial carcinoma.

Results-There were 71 eligible patients who received at least one dose of therapy with 21 of these treated on the combination arm which was closed early because of an excess of venous thrombosis, with 5 episodes of deep venous thrombosis (DVT)and 2 pulmonary emboli. There were three responses observed in that arm (14\%). A total 50 eligible patients were treated on the single agent arm with 3 episodes of DVT and 11 responses (22\%). Response rates were similar in patients with prior chemotherapy ( 7 of $29 ; 24 \%$ ) and those with no prior chemotherapy (4 of 21; $19 \%)$. Two of four patients with clear cell carcinoma responded.

Conclusions-Adding the combination of megestrol acetate and tamoxifen to temsirolimus therapy did not enhance activity and the combination was associated with an excess of venous thrombosis. Temsirolimus activity was preserved in patients with prior adjuvant chemotherapy. These findings will have implications for future trial design.

\section{Keywords}

Temsirolimus; hormonal therapy; megestrol acetate; tamoxifen; endometrial cancer

\section{INTRODUCTION}

Advanced or recurrent endometrial carcinoma is an incurable disease with short overall survival. Standard initial therapy for many years consisted of treatment with medroxyprogesterone acetate or megestrol acetate, and response rates of $15 \%-27 \%$ to progestin-based regimens in chemotherapy-naïve patients have been published by a number of authors $[1,2]$. However the median progression-free survival with such regimens is short, and although low-grade and estrogen or progesterone receptor-positive tumors have higher response rates to endocrine therapy, no reliable predictive factors for benefit have emerged [3]. Currently, most women with advanced disease are initially treated with platinum/taxanebased chemotherapy. Such regimens yield response rates in the range of 50\%, but the median survival remains only about $12-15$ months [4].

As targeted agents began to show promise in a number of different tumor types, interest was focused on the high frequency of aberrations in the PI3K/Akt/mTOR pathway in endometrial cancers [5]. A number of mTOR inhibitors have been tested in endometrial cancer and found to have only modest activity (Table 1). Phase II studies of temsirolimus conducted by the National Cancer Institute of Canada (NCIC) showed a 14\% response rate in chemotherapy-naïve patients and a $4 \%$ response rate in patients with prior chemotherapy [6].

In endometrial cancer cell lines and mouse models, upregulation of PI3K/Akt/mTOR pathway activity is associated with resistance to progestin therapy, and inhibition of the pathway can reverse this resistance [7,8]. Similar observations have been made regarding the association of resistance to tamoxifen and aromatase inhibitors with PI3K/Akt/mTOR pathway activity in breast cancer models. These led to a phase III trial in which breast cancer patients previously treated with a nonsteroidal aromatase inhibitor who were 
randomized to the combination of the mTOR inhibitor, everolimus, plus exemestane had significantly superior progression-free survival compared to those randomized to exemestane alone [9].

We therefore performed a randomized open-label two-stage phase II trial of temsirolimus alone versus the combination of temsirolimus plus a hormonal therapy regimen consisting of alternating megestrol acetate and tamoxifen. This hormonal therapy was chosen based on data published by the Gynecologic Oncology Group (GOG) showing a response rate of 27\% and a median response duration of 28 months with the regimen [2]. While there are no data showing that such a regimen is superior to single agent progestin therapy, regimens including periodic tamoxifen have produced the highest response rates in the GOG experience. Archival tumor tissue was collected and stained for ER, PR, PTEN, and phospho-AKT.

\section{PATIENTS AND METHODS}

\section{Eligibility}

Subjects were required to have measurable endometrial carcinoma (RECIST version 1.0) that was either stage III or IV, or persistent or recurrent after treatment for earlier stage disease. Prior endocrine therapy was prohibited; up to one prior chemotherapy regimen was allowed, but if that regimen were administered in the setting of stage IV disease it was required that the patient have been without evidence of disease at the completion of chemotherapy, and to have had at least six months of progression-free survival since the completion of chemotherapy. Chemoradiotherapy was counted as a chemotherapy regimen. Other eligibility criteria included performance status $0-2$, absolute neutrophil count $\geq 1,500$ / $\mathrm{mcl}$, platelets $\geq 100,000 / \mathrm{mcl}$, total bilirubin sinstitutional upper limit of normal (ULN), AST and alkaline phosphatase $\leq 2.5$ times ULN ( $\leq 5$ times ULN for subjects with liver metastases), creatinine $\leq 1.5 \times \mathrm{ULN}$, fasting cholesterol $\leq 350 \mathrm{mg} / \mathrm{dL}$, fasting triglycerides $\leq$ $400 \mathrm{mg} / \mathrm{dL}$, and albumin $\geq 3.0 \mathrm{mg} / \mathrm{dL}$. Long term corticosteroid use as well as enzymeinducing antiepileptic drugs and other CYP3A4 inducers were prohibited. Patients with known congestive heart failure or a need for oxygen use were excluded, as were those with a history of unprovoked deep venous thrombosis (DVT) or pulmonary embolus (PE), unless maintained on anticoagulation for the duration of the trial. All subjects signed an institutionally approved informed consent including HIPAA authorization. Central review of initial pathologic diagnosis by the GOG Pathology Committee was performed for all cases.

\section{Study Design and Treatment Plan}

Treatment was randomly assigned with equal probability within strata as either single agent temsirolimus at a dose of $25 \mathrm{mg}$ intravenously (IV) weekly or the combination of temsirolimus $25 \mathrm{mg}$ IV weekly plus megestrol acetate $80 \mathrm{mg}$ orally twice a day for 3 weeks alternating with tamoxifen $20 \mathrm{mg}$ orally twice a day for 3 weeks. Randomization was stratified on the basis of prior chemotherapy (yes or no). After closure of the combination arm, accrual to single agent temsirolimus therapy continued. A letter describing the risk of thrombosis was sent to physicians and patients on the combination therapy arm, and they were allowed to choose whether to remain on their current regimen, receive temsirolimus 
alone, or to discontinue protocol-directed therapy. Therapy was to be continued until tumor progression or undue toxicity.

\section{Management of Toxicity}

Administration of subsequent doses of temsirolimus required ANC $>1,000 / \mathrm{mcl}$ and platelets $>100,000 / \mathrm{mcl}$. Temsirolimus was held until these parameters were met and restarted with a $5 \mathrm{mg}$ dose reduction. Tamoxifen and megestrol acetate could be continued while temsirolimus was held. Subjects experiencing a venous thrombotic event were permitted, at the discretion of the investigator, to stay on study with institution of therapeutic anticoagulation. Toxicities requiring cessation of treatment included: grade 2 or higher pneumonitis, requirement for a dose reduction of temsirolimus to less than $15 \mathrm{mg}$, and grade 3 or 4 toxicities requiring over 14 days till recovery to tolerable grade 2 or better.

\section{Evaluation}

Toxicity was graded according to CTCAE version 3.0. Re-evaluation for disease response was performed every six weeks for the first 24 weeks of therapy, and then every 12 weeks. RECIST criteria versions 1.0 were used for assessment of response. The categories of confirmed, complete and partial response were combined to define tumor response. Progression-free survival (PFS) was defined as the time from study entry to death or documentation of disease progression. Patients alive with no evidence of disease progression were censored at the time of their last follow-up. Survival (OS) was defined as the time from study entry to death, regardless of cause; patients last known to be alive were censored at time of last follow-up.

\section{Immunohistochemical Analysis}

Formalin-fixed, paraffin-embedded (FFPE) tissue sections were sent to the GOG Tissue Bank and distributed to the GOG Core Laboratory for Receptors and Targets. Before beginning the experiments, immunostaining protocols and antibody dilution for Estogen Receptor-alpha (ER; Dako, Carpinteria, CA), Progesterone Receptor (PR; Dako, Carpinteria, CA), Progesterone Receptor B (PRB; Cell signaling, Beverly, MA), Ser-473 phospho-AKT XP (pAKT; Cell signaling, Beverly, MA), and PTEN (Millipore, Billerica, MA) were tested and optimized on positive and negative control tissues. Tissue hydration and deparafinization was performed by incubating the slides in three washes of xylene, followed by three washes of ethanol and finally three washes of water. Antigen retrieval was initiated by microwaving the slides at sub-boiling temperatures in $10 \mathrm{mM}$ of sodium citrate buffer with pH 6.0 for 10min for ER and pAKT, and 20 min for PR, PRB and PTEN. The slides were then cooled at room temperature for $30 \mathrm{~min}$. Quenching of endogenous peroxidases was achieved by incubating the sections in $3 \%$ hydrogen peroxide for $10 \mathrm{~min}$. The sections were then rinsed and blocked using 5\% normal goat serum for $30 \mathrm{~min}$ (PR, PRB and PTEN) to 1 hour (pAKT), followed by overnight incubation at $4{ }^{\circ} \mathrm{C}$ in the primary antibodies listed above. Tissues were then rinsed and incubated for $30 \mathrm{~min}$ in biotinylated goat anti-mouse antibody for PR, goat anti-rabbit for PRB and PTEN, mouse DAKO EnVision ${ }^{\mathrm{TM}}$ HRP System for ER, and SignalsStain ${ }^{\mathrm{TM}}$ Boost Detection Reagent for pAKT. After rinsing, the sections were incubated in DAB chromogen substrate, counterstained 
using hematoxylin, dehydrated using three washes of ethanol and xylene, and mounted with coverslips.

The slides were reviewed independently by two investigators (KL, HR) blinded to the treatment regimen and clinical outcome. The proportion and intensity of staining was recorded as $0-100 \%$ and 0 to $4+$, respectively. These values were multiplied together to determine a modified H-score. Discrepancies of more than $20 \%$ in the modified $\mathrm{H}$-score values were adjudicated by a member of the GOG Pathology Committee, Dr Meenakshi Singh. The staining was further reviewed by RZ; no significant differences in staining or interpretation were found. Because of the variable age of the slides, it was assumed that there would be general, but variable, loss of immunoreactivity. Therefore, although modified $\mathrm{H}$-scores were calculated for most of the analyses performed, any level of staining for any of the stains was considered positive and results were simply dichotomized as positive or negative. However, the continuous version of the modified $\mathrm{H}$ score was used for identifying correlation between markers and for the proportional hazards model.

\section{Statistical Considerations}

The primary endpoint of the trial was clinical complete or partial response. The first stage of the trial was to be stopped after accrual of 21 patients to each arm, with the number of responses required to go to the second stage dependent on how many patients had prior chemotherapy.

A two-stage conditional stratified phase II trial as proposed by London and Chang was used which utilizes the marginal number of responses across all populations while factoring differing probabilities of response within each population [10]. Conditioned on the realized sample size in each stratum, the probability mass function for $\mathrm{R}_{1}$ and $\mathrm{R}_{2}$ corresponding to the responses produced in stages 1 and 2 can be found with:

$$
P\left(R_{j}=r_{j}\right)=\sum_{r_{1 j}+\cdots+r_{k j}=r_{j} i=1} \prod^{2}\left(\begin{array}{c}
n_{i j} \\
r_{i j}
\end{array}\right) p_{i}^{r_{i j}}\left(1-p_{i}\right)^{n_{i j}-r_{i j}}, j=1,2 .
$$

where $i$ indexes the $\mathrm{k}=2$ important stratification levels under consideration and $j$ indexes the stage of accrual. The distribution of $R_{j}$ depends on the probabilities of response, $p_{i}$, within each stratum. Stratum 1 corresponded with those patients who had never been treated with chemotherapy whereas stratum 2 corresponded with those patients who had prior chemotherapy. The null hypothesis of no treatment effect is $\mathrm{H}_{0}$ : $\mathrm{p}_{1}=0.20$ and $\mathrm{p}_{2}=0.10$. Under the alternative hypothesis of $\mathrm{H}_{1}: \mathrm{p}_{1}=0.40$ and $\mathrm{p}_{2}=0.30$, the following design will limit the probability of type I error to 0.06 and type II to 0.10 . A confidence interval for the true response rate, adjusted for multistage design when appropriate, is reported for each arm [11].

Translational research endpoints were analyzed in an exploratory manner and were not considered when determining the sample size of this trial. Beyond basic summary statistics, the Spearman rank-order correlation statistic was used to assess correlation between biomarkers [12]. The Jonckheere-Terpstra test was used to test the association of biomarker 
modified H-score with increasing tumor grade [13]. The modified H-score was collapsed into two categories for some analyses; 0 (no expression) and greater than 0 (any expression). Fisher's exact test was used to test 2 by 2 associations between biomarker expression and RECIST response [14]. A Cox proportional hazards model was fit for each biomarker to assess the association of modified $\mathrm{H}$-score with progression-free and overall survival [15]. Kaplan-Meier estimates of the distribution of survival and progression-free survival times were plotted by treatment arm and by biomarker expression combined with treatment arm [16].

\section{RESULTS}

Seventy-three patients were registered to this trial between 9/29/08 and 11/22/10. Two were excluded from analysis; one did not meet eligibility requirements after central review and one never received any protocol therapy. Figure 1 (supplemental) shows the outcomes of all patients registered to the trial. Patient characteristics are shown in Table 2. At the time of writing two patients on the single agent temsirolimus arm were still receiving therapy at 30 and 45 months from enrollment.

\section{Adverse Events}

On 10/19/09 the trial was suspended and the combination arm was permanently closed to accrual because an excess of venous thromboses was noted. At this time 22 patients had been treated on combination therapy (one of whom was ineligible), and there had been five events of deep venous thrombosis (DVT), two pulmonary emboli, one myocardial infarction, and one sudden death. At that time point there had been no thrombotic events reported among the 21 patients on the single agent temsirolimus arm; subsequently three patients receiving single agent temsirolimus experienced a DVT. The p-value for Fisher's exact test of an association between treatment arm and thrombotic events at the time the trial was closed is 0.048 .

Other key adverse events are shown in Table 3 (supplementary), and are generally those expected from mTOR inhibitors. The most common side effects overall included low-grade myelosuppression, rash, fatigue, hyperlipidemia, edema, pneumonitis, and gastrointestinal toxicities including nausea, diarrhea, anorexia and mucositis. On the single agent temsirolimus arm 11 patients (22\%) came off study treatment for toxicity which mandated cessation of study therapy per protocol, and $5(10 \%)$ of patients wished to stop therapy in absence of progression or protocol-specified toxicity. On the combination arm study treatment was discontinued in six patients $(28.6 \%)$ for protocol-specified toxicity and in one (4.8\%) for patient preference. Seven patients were removed from protocol therapy because of pneumonitis, (two on the combination arm and five on the single agent arm, including one who died). Two patients, one on each arm, came off study for edema. Twenty-two percent of the women treated on this trial $(n=16)$ were seventy years or older. They did not have an overall excess of toxicity, although fatigue may have been more common. Grade $\geq 3$ fatigue was seen in $3 / 55(5.4 \%$, all grade 3$)$ of women younger than 70 and $2 / 16(12.5 \%$, one grade 3 and one grade 4$)$ of women aged 70 or older. For mucositis, 2/16 (6\%) of women aged $\geq$ 70 experienced grade 2 symptoms versus $7 / 55(12.7 \%)$ of younger women. 


\section{Responses}

At the time the combination arm was closed, $3 / 21$ (14\%; 94\% confidence interval 3\%-36\%) eligible patients had a partial response, which would have met the criteria to proceed to second stage. Histologic subtypes of the responding tumors were: one grade 1 endometrioid, one grade 3 endometrioid, and one grade 2 adenocarcinoma, not otherwise specified. No further patient on this arm subsequently met criteria for a response. Six of the first 21 patients in the single agent temsirolimus arm had a response, and a total of 50 eligible patients were treated on that arm. There were 11 responses $(22 \%$; $94 \%$ confidence interval $11 \%--52 \%$ ), three complete responses (CR) and eight partial responses (PR). Histologic subtypes of responding tumors were: one grade 1 endometrioid, three grade 2 endometrioid, two grade 3 endometrioid, one mixed, two serous, and two clear cell. The median duration of response was 8.5 months. Twenty-six patients had stable disease (SD) as their best response, with a median duration of 7.9 months. Breakdown of responses by prior therapy is seen in Table 4. On the single agent temsirolimus arm, the response rate was $24 \%$ for patients with prior chemotherapy and $19 \%$ for those with no prior therapy. Progression-free and overall survival by arm are shown in Figure 2a. Median progression-free and overall survival (both arms combined) were 4.9 months and 10.8 months for those with prior chemotherapy versus 8.2 months and 20.7 months for those without (Figure 2b).

Information on use of concomitant medications was collected at baseline. Five patients were on metformin. None of the three patients on metformin who were randomized to single agent temsirolimus had a major response (1 progressive disease (PD), 2 SD), and one of the two on the combination of temsirolimus plus hormonal therapy responded (1PR, $1 \mathrm{PD}$ ). Seven patients were receiving angiotensin converting enzyme (ACE) inhibitors at time of study registration; none developed pneumonitis. Information on changes in medications during the trial was not collected.

\section{Immunohistochemistry}

Of the 73 patients enrolled in the study, three were deemed ineligible, seven did not have primary tissue available for study, and five had an insufficient number of FFPE slides to complete the analysis. Of the 58 remaining cases, two were judged to have no remaining tumor tissue on the submitted slides, leaving a total of 56 patients included in the analysis: 36 on single agent temsirolimus and 20 on combination therapy. After immunostaining was performed, 30 cases were found to be positive for PR, 35 for PRB, 20 for ER, 11 for pAKT, and 30 for PTEN (Figure 4).

Of the 11 cases that stained for pAKT (regardless of PTEN status), nine were treated on single agent temsirolimus, and four achieved a clinical response. Neither of the two patients staining for pAKT responded in the combination arm. Response data by pAKT is shown in Table 5 (supplementary). The association of pAKT with OS for each treatment arm is shown in Figure 3a. Twenty-four tumors were negative for pAKT and positive for PTEN. Of these cases, five patients had a clinical response. Twenty-one cases were negative for both pAKT and PTEN. Only two responses were observed in this group. There was no statistically significant association between pAKT or PTEN expression and response, but response 
estimates are consistent with fewer responders among those whose tumors were negative for both pAKT and PTEN.

There was a statistically significant inverse correlation between PR H-score and tumor grade; there was no significant association with grade for any of the other biomarkers. Both of the responding tumors on the combination therapy arm showed positive staining for ER, $\mathrm{PR}$ and PRB. The response to temsirolimus as a single agent did not significantly vary by ER, PR or PRB expression status (ER- 24\%, ER+ 27\%; PR- 27\%, PR+ 24\%; PRB- 23\%, $\mathrm{PRB}+26 \%$ ). The association of ER with OS for each treatment arm is shown in Figure 3b. While it was not statistically significant, the hazard ratio (HR) estimates for a 100 unit increase in modified ER H-score suggest a larger relative survival benefit in those with higher ER H-scores compared with lower ER H-scores (HR=0.38; Wald 95\% confidence interval: 0.11 to 1.25 ) in the hormonal arm as compared with that in the non-hormonal arm (HR=0.71; Wald 95\% confidence interval: 0.35 to 1.45 ). This is consistent with the role of ER as a potential predictive marker for response to hormone therapy [3]. Additionally, ER and PR were positively correlated with each other.

\section{DISCUSSION}

This trial confirms the activity level of single agent temsirolimus in women with endometrial cancer. The response rate in this group was $22 \%$, which is very similar to that reported by Oza et al (24\% investigator-reported; $14 \%$ on independent radiology review) for chemotherapy naïve patients [17] and considerably better than Oza et al observed in pretreated patients (4\%). This suggests that patients who would have met eligibility for our trial, with prior adjuvant chemotherapy or at least six-month progression free interval since prior chemotherapy in the setting of advanced disease, are more likely to respond to temsirolimus than the average patient receiving second-line therapy for advanced disease. Similar results have been found for second-line platinum-based therapy, with a higher likelihood of response associated with a longer platinum-free interval. [18] We also confirmed that responses are observed in all histologic subtypes of endometrial cancer. The responses seen in two of four women with clear cell carcinoma are very encouraging for benefit in women with that rare histology. As in other trials of mTOR inhibitors, there were a few patients with prolonged benefit, including one who has been on study for 45 months. The two women still on treatment at the time of data analysis both had tumors of endometrioid histology (one grade1, and one grade 2).

We saw no evidence of increased benefit with the addition of a hormonal regimen consisting of alternating megestrol acetate and tamoxifen to temsirolimus. This is not simply because of increased toxicity. There was no trend to an improved response rate with the combination. This is different from preliminary results reported by Slomovitz et al, who described no responses to the mTOR inhibitor everolimus when it was given as a single agent to women with progressive or recurrent endometrial cancer [19], whereas a successor trial in the same population using the combination of letrozole plus everolimus produced a response rate of $31 \%$ [20]. A study with letrozole alone in women with chemotherapy-naïve advanced endometrial cancer yielded a response rate of only $9.4 \%$ [21]. It is very possible that there are mechanistic differences between the interactions of mTOR inhibitors with progestins 
versus aromatase inhibitors. However, while everolimus is approved for use in breast cancer specifically in combination with an aromatase inhibitor, benefits to the addition of mTOR inhibitors to tamoxifen have also been observed in two smaller randomized trials, suggesting the effect in breast cancer may not be specific to only one hormonal agent $[22,23]$. Given that our results showed a trend towards benefit to combination therapy in women with ER positive tumors, we would suggest any future trials of the combination of mTOR inhibitors and hormonal agents in women with endometrial cancer be limited to those with ER positive tumors.

The increased rate of thrombosis that we observed is likely multifactorial. First, the regimen of tamoxifen alternating with megestrol acetate is fairly thrombogenic by itself in women with endometrial cancer. Four pulmonary emboli, 2 deep venous thromboses and one stroke were reported among 56 women in the original publication (12.5\% rate of thrombosis) [2]. This is higher than the $5 \%$ rate of venous thrombosis reported on prior GOG trials using megestrol acetate alone [1] (which is similar to the 5\% rate of venous thrombosis reported in studies using megestrol acetate for appetite stimulation in women with cancer in general) and was likely further increased by the insertion of central venous access devices for many women in our study [24]. At least two of the reported thromboses appeared to be catheterrelated. Prophylactic anticoagulation should be considered in women with advanced endometrial cancer treated on a combination of megestrol acetate and tamoxifen, particularly if a central venous access device is in place. The thrombotic risk was likely exacerbated by the edema that is a class effect of mTOR inhibitors, and may be related to inhibition of lymphangiogenesis [25]. Eleven of 21 patients on the combination arm and 18 of 50 patients on the single agent temsirolimus arm reported grade 1 or greater edema and edema per se was the reason for discontinuation of therapy in two patients. In addition, use of temsirolimus can result in compensatory upregulation of phospho-AKT, which can upregulate tissue factor. Interestingly, the TAMRAD trial, which randomized women with metastatic breast cancer to tamoxifen alone or the combination of tamoxifen and everolimus did not report an excess of venous thromboses in the combination arm; there were 2 episodes of DVT and one PE reported in the 57 women on tamoxifen alone and three episodes of DVT described in the 54 women receiving combination therapy [22]. It is possible that megestrol acetate is an important contributor to the risk of thrombosis or that women with metastatic breast cancer, who do not tend to have pelvic disease, are less prone to venous thrombosis than women with metastatic endometrial cancer.

Other toxicities on this trial were as reported in previous studies of mTOR inhibitors. Pneumonitis remained problematic, and was the probable cause of one death as well as resulting in discontinuation of protocol therapy for seven (10\%) women. It has been reported that ACE inhibitor therapy is protective against pneumonia [26] and radiation pneumonitis [27]; none of the patients who developed pneumonitis were taking ACE inhibitors at the time of study entry, but the numbers were small. Patients over the age of 70 years did not have more toxicity in general, although severe fatigue appeared more common in this age group.

We did not see any differences in response rates in women who were receiving metformin at baseline in either the single agent of the combination arm. However, this does not refute the 
hypothesis that metformin treatment may be associated with better outcomes for endometrial cancer patients, as we did not collect information on concomitant medications throughout the study. Temsirolimus can cause hyperglycemia and we do not know which patients might have started metformin during the trial.

GOG 248 was not powered to test translational research outcomes. We hypothesized that tumors with loss of PTEN would exhibit activation of AKT as demonstrated by phosphorylation at Ser-473 and vice versa, that is positive PTEN staining would be linked to decreased pAKT. However, we observed that while the expected negative correlation between PTEN and pAKT was present in a subset of tumors (Figure 4), other tumors were positive or negative for both factors. Possible reasons for this are that PTEN mutations in endometrial cancer do not always result in loss of PTEN immunohistochemical staining and there can be other PI3K pathway alterations, such as PI3K or AKT mutation that result in increased pAKT [28]. Other small studies have not shown any correlation between tumor immunohistochemical markers and response to mTOR inhibitors in endometrial cancer. Oza et al stained tissue for 58 of the 62 patients on their Temsirolimus trials for PTEN, pAKT, pS6, and pmTOR, and found no correlation between response or progression and any marker [29]. Tredan et al reported on immunohistochemical analyses of tumors from 36 of the 44 patients on the Endorad trial of single agent everolimus, and found that patient outcome was not predicted by pAKT or PTEN expression, or by immunohistochemical results of staining for ER/PR, HER2, LKB1, PI3K, 4E-BP1, p4E-BP1, or S6RP [30]. Our findings of a trend towards pAKT status as a predictor of response to temsirolimus is supported by preclinical work, [31] but may be due to chance as our numbers were small. The slides on this trial were not all freshly cut; sites could provide blocks or unstained slides. We dichotomized stain results (rather than using an $\mathrm{H}$-score) to help compensate for possibly decreased stain intensity, but there are likely some false-negative which weakens conclusions about any possible associations. However, our findings are very consistent with the premise that ER is likely the most predictive marker for response and survival with temsirolimus combined with hormonal therapy. As expected, hormone receptor status does not appear to be predictive of response to temsirolimus alone. Further work to find predictors of response to mTOR inhibitors is clearly needed; KRAS mutations have been suggested to be associated with resistance to $\mathrm{mTOR}$ inhibitor therapy, and this would be an interesting direction to explore [32].

Interestingly, the overall survival for women with no prior chemotherapy on this study was 20.7 months, which well exceeds that seen in most prior front-line trials for endometrial cancer, and at the very least suggests that the use of an mTOR inhibitor prior to chemotherapy does not provide any survival disadvantage. The GOG reported median overall survivals of 15.3 months with Taxol/doxorubicin/cisplatin [4] and 14 months with the hormonal regimen on tamoxifen alternating with megestrol acetate [2] in chemotherapynaïve populations. However, it seems likely that optimal use of mTOR inhibitor therapy for most women with endometrial carcinoma will be in combination with other agents. Results of GOG 86P, which has an arm testing the combination of carboplatin, paclitaxel, and temsirolimus followed by maintenance temsirolimus, should be available soon. 


\section{Supplementary Material}

Refer to Web version on PubMed Central for supplementary material.

\section{Acknowledgments}

The authors would like to acknowledge Dr. Meenakshi Singh from Stonybrook University Medical Center, as the GOG Core Laboratory Pathologist, as well as Dr. Yuping Zhang at the University of Iowa for assistance with these assays.

This study was supported by National Cancer Institute grants to the Gynecologic Oncology Group (GOG) Administrative Office and the GOG Tissue Bank (CA 27469), the GOG Statistical and Data Center (CA 37517).

The following Gynecologic Oncology Group member institutions participated in the primary treatment studies: Duke University Medical Center, Abington Memorial Hospital, Walter Reed Army Medical Center, University of Minnesota Medical School, Colorado Gynecologic Oncology Group P.C., University of Cincinnati, University of North Carolina School of Medicine, University of Iowa Hospitals and Clinics, University of Texas Southwestern Medical Center at Dallas, Indiana University School of Medicine, University of California Medical Center at Irvine, Rush-Presbyterian-St. Luke's Medical Center, University of New Mexico Health Sciences Center, Stony Brook University Hospital, Memorial Sloan-Kettering Cancer Center, Cooper Hospital/University Medical Center, University of Massachusetts Memorial Health Center, Fox Chase Cancer Center, Women's Cancer Center, University of Oklahoma, University of Virginia Health Sciences Center, University of Chicago, Case Western Research University, University of Wisconsin Hospital, Women and Infants Hospital, The Hospital of Central Conn at New Britain General, Georgia Core, GYN Oncology of West Michigan, PLLC and Community Clinical Oncology Program.

\section{References}

1. Thigpen JT, Brady MF, Alvarez RD, Adelson MD, Homesley HD, Manetta A, et al. Oral medroxyprogesterone acetate in the treatment of advanced or recurrent endometrial carcinoma: a dose-response study by the Gynecologic Oncology Group. J Clin Oncol. 1999; 17:1736-44. [PubMed: 10561210]

2. Fiorica JV, Brunetto VL, Hanjani P, Lentz SS, Mannel R, Andersen W. Phase II trial of alternating courses of megestrol acetate and tamoxifen in advanced endometrial carcinoma: a Gynecologic Oncology Group study. Gynecol Oncol. 2004; 92:10-4. [PubMed: 14751131]

3. Singh M, Zaino RJ, Filiaci VJ, Leslie KK. Relationship of estrogen and progesterone receptors to clinical outcome in metastatic endometrial carcinoma: a Gynecologic Oncology Group Study. Gynecol Oncol. 2007; 106:325-33. [PubMed: 17532033]

4. Fleming GF, Brunetto VL, Cella D, Look KY, Reid GC, Munkarah AR, et al. Phase III trial of doxorubicin plus cisplatin with or without paclitaxel plus filgrastim in advanced endometrial carcinoma: a Gynecologic Oncology Group Study. J Clin Oncol. 2004; 22:2159-66. [PubMed: 15169803]

5. Weigelt B, Banerjee S. Molecular targets and targeted therapeutics in endometrial cancer. Curr Opin Oncol. 2012; 24:554. [PubMed: 22581357]

6. Diaz-Padilla I, Duran I, Clarke BA, Oza AM. Biologic rationale and clinical activity of mTOR inhibitors in gynecological cancer. Cancer Treat Rev. 2012; 38:767-75. [PubMed: 22381585]

7. Gu C, Zhang Z, Yu Y, Liu Zhao F, Yin L, Feng Y, Chen X. Inhibiting the PI3K/Akt pathway reversed progestin resistance in endometrial cancer. Cancer Sci. 2011; 102:557-64. [PubMed: 21205080]

8. Pant A, Lee, Lu Z, Rueda BR, Schink J, Kim JJ. Inhibition of AKT with the orally active allosteric AKT inhibitor, MK-2206, sensitizes endometrial cancer cells to progestin. PLoS One. 2012; 7:e41593. [PubMed: 22911820]

9. Baselga J, Campone M, Piccart M, Burris HA 3rd, Rugo HS, Sahmoud T, et al. Everolimus in postmenopausal hormone-receptor-positive advanced breast cancer. N Engl J Med. 2012; 366:520 29. [PubMed: 22149876]

10. London WB, Chang MN. One- and two-stage designs for stratified phase II clinical trials. Stat Med. 2005; 24:2597-611. [PubMed: 16118809] 
11. Atkinson EN, Brown BW. Confidence limits for probability of response in multistage phase II clinical trials. Biometrics. 1985; 41:741-44. [PubMed: 4074823]

12. Conover, WJ. Practical Nonparametric Statistics. 3. New York: John Wiley \& Sons; 1998.

13. Pirie, W. Jonckheere Tests for Ordered Alternatives. In: Kotz, S.; Johnson, NL., editors. Encyclopedia of Statistical Sciences. Vol. 4. New York: John Wiley \& Sons; 1983. p. 315-318.

14. Agresti A. A survey of exact inference for contingency tables. Statist Sci. 1992; 7:131-153.

15. Cox DR. Regression Models and Life Tables. J R Stat Soc, Series B. 1972; 20:187-20.

16. Kaplan EL, Meier P. Nonparametric estimation from incomplete observations. J Amer Statist Assn. 1958; 53:457-81.

17. Oza AM, Elit L, Tsao MS, Kamel-Reid S, Biagi J, Provencher DM, et al. Phase II study of temsirolimus in women with recurrent or metastatic endometrial cancer: a trial of the NCIC Clinical Trials Group. J Clin Oncol. 2011; 29:3278-85. [PubMed: 21788564]

18. Nagao S, Nishio S, Michimae H, Tanabe H, Okada S, Otsuki T, et al. Applicability of the concept of "platinum sensitivity" to recurrent endometrial cancer: The SGSG-012/GOTIC-004/Intergroup study. Gynecol Oncol. 2013; 131:567-73. [PubMed: 24076450]

19. Slomovitz BM, Lu KH, Johnston T, Coleman RL, Munsell M, Broaddus RR, et al. A phase 2 study of the oral mammalian target of rapamycin inhibitor, everolimus, in patients with recurrent endometrial carcinoma. Cancer. 2010; 116:5415-19. [PubMed: 20681032]

20. Slomovitz BM, Soliman P, Levenback C, Brown J, Wolf J, Schmeler K, et al. Phase I/II multi-site clinical trials: Brief Communications on uterine cancer including sarcoma. Proc 14th Biennial Meeting of the International Gynecologic Cancer Society, IGCS. 2012; 22:E243. (abstr).

21. Ma BB, Oza A, Eisenhauer E, Stanimir G, Carey M, Chapman W, et al. The activity of letrozole in patients with advanced or recurrent endometrial cancer and correlation with biological markers--a study of the National Cancer Institute of Canada Clinical Trials Group. Int J Gynecol Cancer. 2004; 14:650-58. [PubMed: 15304161]

22. Bachelot T, Bourgier C, Cropet C, Ray-Coquard I, Ferrero JM, Freyer G, et al. Randomized phase II trial of everolimus in combination with tamoxifen in patients with hormone receptor-positive, human epidermal growth factor receptor 2-negative metastatic breast cancer with prior exposure to aromatase inhibitors: a GINECO study. J Clin Oncol. 2012; 30:2718-24. [PubMed: 22565002]

23. Bhattacharyya GS, Biswas J, Singh JK, Singh M, Govindbabu K, Ranade AAM, et al. Reversal of tamoxifen resistance (hormone resistance) by addition of sirolimus (mTOR inhibitor) in metastatic breast cancer. Eur J Cancer. 2011; 47:9.

24. Loprinzi CL, Kugler JW, Sloan JA, Mailliard JA, Krook JE, Wilwerding MB, et al. Randomized comparison of megestrol acetate versus dexamethasone versus fluoxymesterone for the treatment of cancer anorexia/cachexia. J Clin Oncol. 1999; 17:3299-306. [PubMed: 10506633]

25. Huber S, Bruns CJ, Schmid G, Hermann PC, Conrad C, Niess H, et al. Inhibition of the mammalian target of rapamycin impedes lymphangiogenesis. Kidney Int. 2007; 71:771-77. [PubMed: 17299523]

26. Sekizawa K, Matsui T, Nakagawa T, Nakayama K, Sasaki H. ACE inhibitors and pneumonia. Lancet. 1998; 352:1069. [PubMed: 9759784]

27. Kharofa J, Cohen EP, Tomic R, Xiang Q, Gore E. Decreased risk of radiation pneumonitis with incidental concurrent use of angiotensin-converting enzyme inhibitors and thoracic radiation therapy. Int J Radiat Oncol Biol Phys. 2012; 84:238-43. [PubMed: 22300564]

28. Djordjevic B, Hennessy BT, Li J, Barkoh BA, Luthra R, Mills GB, et al. Clinical assessment of PTEN loss in endometrial carcinoma: immunohistochemistry outperforms gene sequencing. Mod Pathol. 2012; 25:699-708. [PubMed: 22301702]

29. Oza AM, Poveda A, Clamp AR, Pignata S, Scambia G, Del Campo JM, et al. A randomized phase II (RP2) trial of ridaforolimus (R) compared with progestin (P) or chemotherapy (C) in female adult patients with advanced endometrial carcinoma. J Clin Oncol. 2011; 29 ASCO 5009 (abstr).

30. Tredan O, Treilleux I, Wang Q, Gane N, Pissaloux D, Bonnin N, et al. Predicting everolimus treatment efficacy in patients with advanced endometrial carcinoma: a GINECO group study. Target Oncol. 2012 Dec 13. Epub ahead of print.

31. Yang S, Xiao X, Meng X, Leslie KK. A mechanism for synergy with combined mTOR and PI3 kinase inhibitors. PLoS One. 2011; 6:e26343. [PubMed: 22039466] 
32. Myers AP. New strategies in endometrial cancer: Targeting the PI3K/mTOR pathway - - The devil is in the details. Clin Cancer Res. 2013; 19:5264-74. [PubMed: 24089439]

33. Ray-Coquard I, Plantade A, Petit T, Lebrun-Jezekova D, Lavau-Denes S, Cretin J, et al. The ENDORAD trial: RAD001 (everolimus) monotherapy as second line or third treatment of advanced endometrial carcinoma: a phase II trial of GINECO group. EJC Supplements. 2009; 7:1. (Abstr\#8046) (abstr).

34. Colombo N, McMeekin DS, Schwartz PE, Sessa C, Gehrig PA, Holloway R, et al. Ridaforolimus as a single agent in advanced endometrial cancer: Results of a single-arm phase 2 trial. $\mathrm{Br} \mathrm{J}$ Cancer. 2013; 108:1021-26. [PubMed: 23403817]

35. Mackay H, Welch S, Tsao MS, Biagi JJ, Elit L, Ghatage P, et al. A phase II study of oral ridaforolimus inpatients with metastatic and/or locally advanced recurrent endometrial cancer: NCIC CTG IND 192. J Clin Oncol. 2011; 29 ASCO 5013 (abstr). 


\section{Higlights}

\section{Bullet Points}

- The combination of hormone therapy plus temsirolimus did not improve response rates compared to temsirolimus alone.

- The combination of therapy with megestrol acetate/tamoxifen plus temsirolimus resulted in a $33 \%$ rate of venous thrombosis

- Two of four patientss with clear cell carcinoma of the endometrium responded to temsirolimus 

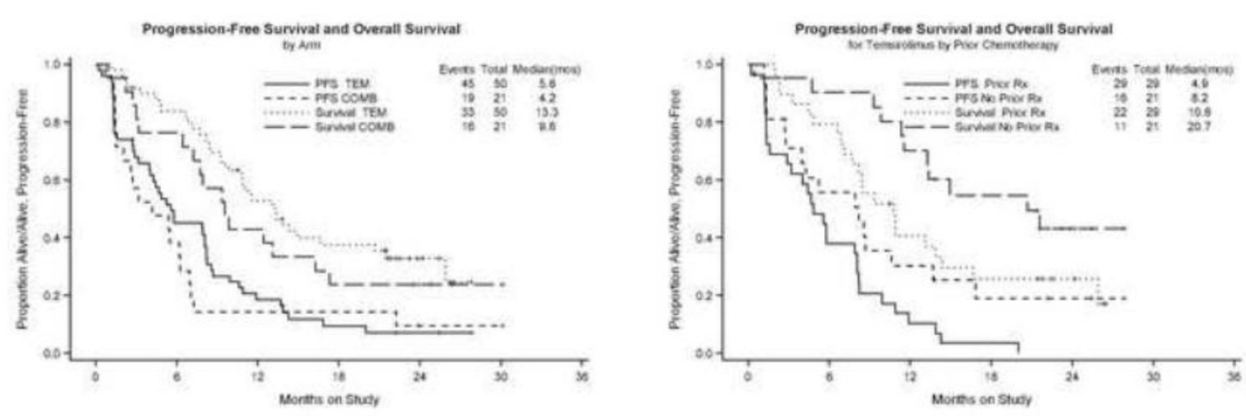

Figure 2.

Figure 2a. PFS and OS by arm

Figure $2 \mathrm{~b}$. PFS and OS by prior therapy 

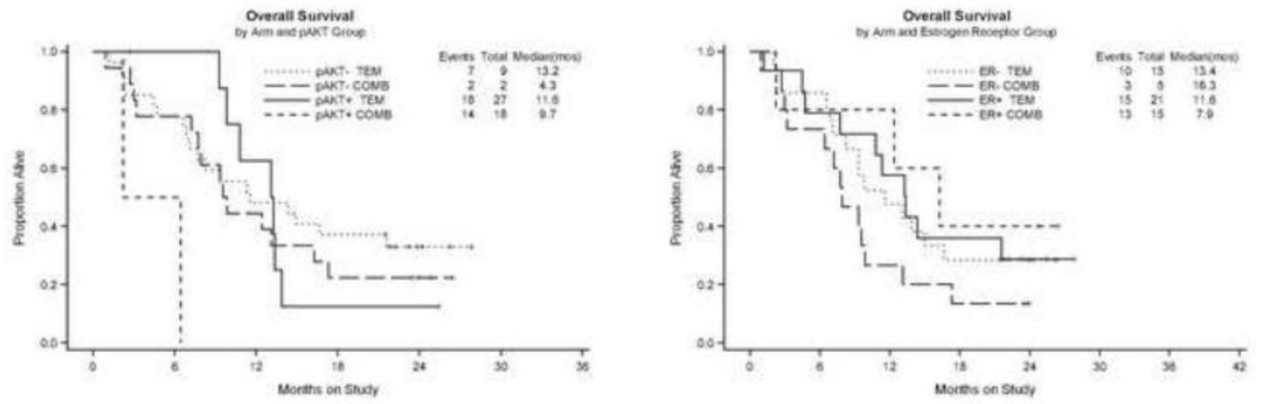

Figure 3.

Figure 3a. OS by pAKT

Figure $3 \mathrm{~b}$ OS by ER 


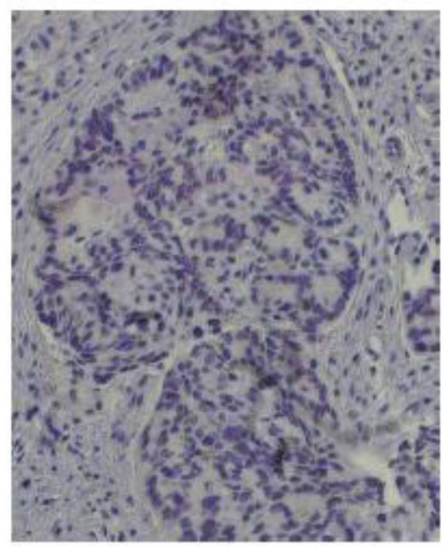

A. PTEN negative

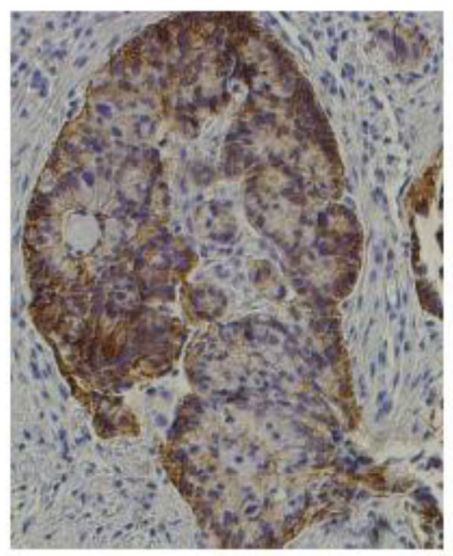

B. Phospho-Ser-473 AKT (pAKT) positive

Figure 4.

Immunohistochemistry for PTEN and pAKT

Immunostaining for PTEN and pAKT in the same tumor, demonstrating the expected inverse correlation between loss of PTEN and activation of AKT. 
Table 1

Trials of Single-Agent mTOR Inhibitors in Endometrial Cancer

\begin{tabular}{llcc}
\hline Author & Agent & Prior Chemotherapy Regimens & RR \\
\hline Oza et al (17) & Temsirolimus & None & $14 \%$ \\
Oza et al (17) & Temsirolimus & $1-2$ & $4 \%$ \\
Slomovitz et al (19) & Everolimus & $1-2$ & 0 \\
Ray-Coquard et al (33) & Everolimus & $1-2$ & $6.8 \%$ \\
Colombo et al (34) & Ridaforolimus IV & $0-2$ & $11 \%$ \\
Mackay et al (35) & Ridaforolimus PO & Adjuvant only & 7.7 \\
Oza et al (29) & Ridaforolimus & $1-2$ & 0 \\
\hline
\end{tabular}

$\mathrm{RR}=$ response rate; $\mathrm{IV}=$ intravenous; $\mathrm{PO}=$ per os (orally) 


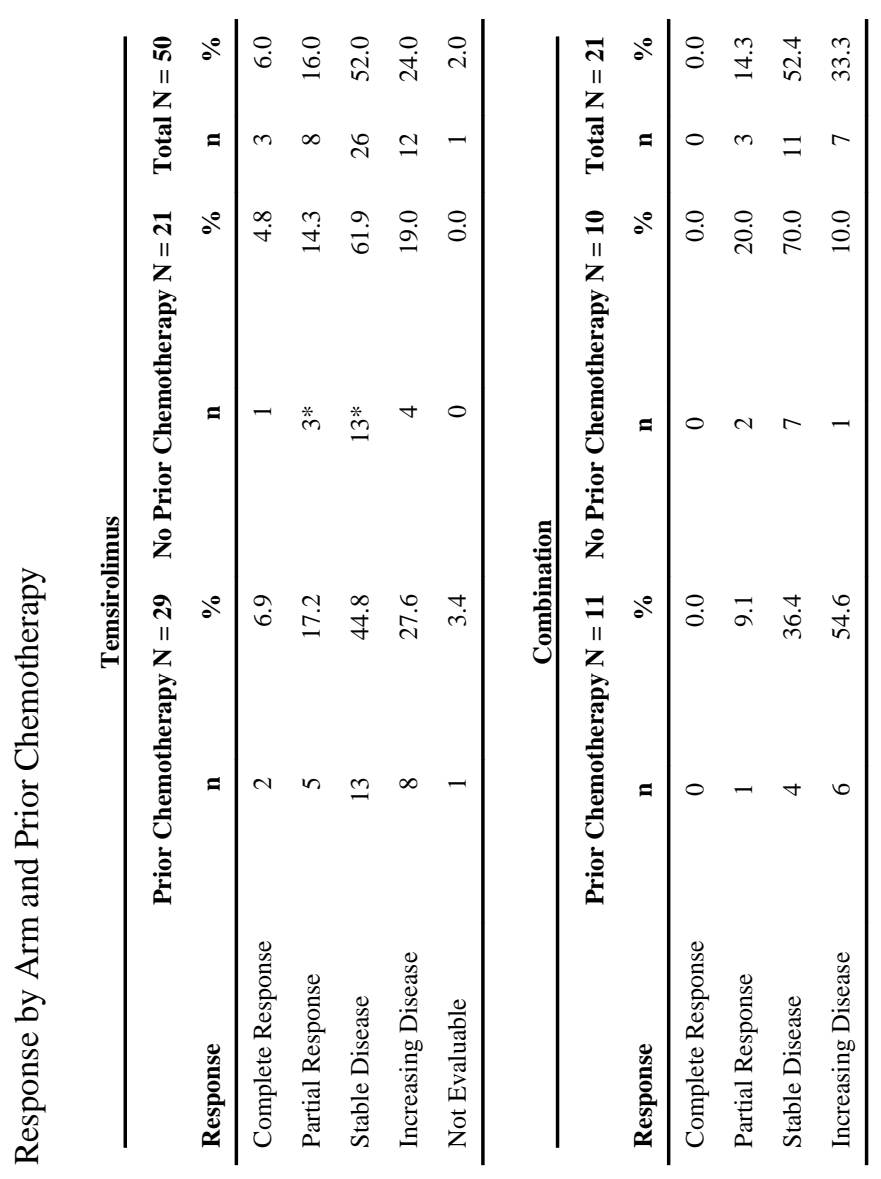

